\title{
Low-density Lipoprotein-Cholesterol Lowering Strategies for Prevention of Atherosclerotic Cardiovascular Disease: Focus on siRNA Treatment Targeting PCSK9 (Inclisiran)
}

\author{
David Sinning ${ }^{1} \cdot$ Ulf Landmesser $^{1,2,3}$ \\ Accepted: 22 September 2020 / Published online: 21 October 2020 \\ (C) The Author(s) 2020
}

\begin{abstract}
Purpose of Review The aim of low-density lipoprotein-cholesterol (LDL-C) lowering therapies is to safely achieve a consistent and long-term reduction in exposure of the vasculature to atherogenic lipoproteins in order to reduce the risk of atherosclerotic cardiovascular (CV) disease and the associated CV events, such as myocardial infarctions and ischemic strokes. This review summarizes the concept and clinical development of a novel molecular approach to efficiently lower LDL-C, a synthetic small interfering ribonucleic acid (siRNA) - inclisiran — directed against proprotein convertase subtilisin-kexin type 9 (PCSK9).

Recent Findings The understanding of genes regulating atherogenic lipoproteins and their causal role in the development of atherosclerotic $\mathrm{CV}$ disease has substantially advanced over the past years. This has opened the possibility for development of molecular therapies targeting these atherogenic lipoproteins, in particular by RNA-targeted treatment approaches. The most advanced clinical development program is the siRNA-treatment targeting PCSK9 (inclisiran), involving more than 4000 patients in clinical studies. Phase 1 and 2 studies have identified the dose of $300 \mathrm{mg}$ inclisiran for efficient LDL-C lowering. Most recently, three phase 3 studies demonstrated that a regimen of inclisiran every 6 months was feasible and reduced LDL-C by approximately $50 \%$ in patients at high or very high CV risk or with familial hypercholesterolemia. Adverse events were similar in the inclisiran and the placebo groups, except for more frequent transient injection site reactions with inclisiran than with placebo. Summary siRNA therapy targeting PCSK9 (inclisiran) applied twice a year efficiently reduced LDL-C by approximately 50\% and was safe in recent phase 3 studies. The effects of this treatment on CV outcome are currently further assessed in a large ongoing $\mathrm{CV}$ outcome trial.
\end{abstract}

Keywords siRNA $\cdot$ Hypercholesterolemia $\cdot$ LDL-C $\cdot$ PCSK $9 \cdot$ Coronary disease

This article is part of the Topical Collection on New Therapies for Cardiovascular Disease

Ulf Landmesser

ulf.landmesser@charite.de

David Sinning

david.sinning@charite.de

1 Department of Cardiology, Charité - University Medicine Berlin (Campus Benjamin Franklin), Berlin, Germany

2 Berlin Institute of Health (BIH), Hindenburgdamm 30, 12203 Berlin, Germany

3 German Centre for Cardiovascular Research (DZHK), Partner Site Berlin, Berlin, Germany

\section{Introduction}

Improved preventive and therapeutic strategies for atherosclerotic cardiovascular disease (ASCVD) have led to a decrease in age-adjusted mortality from ASCVD [1, 2]. ASCVD remains, however, a leading cause of morbidity and mortality globally [3-5]. In Europe, ASCVD is considered to cause more than 4 million deaths annually and is thought to be accountable for up to $45 \%$ of all deaths [4].

Dyslipidemia represents a particular relevant risk factor for ASCVD: in the INTERHEART case-control study with 27.098 participants, an elevated apolipoprotein B/apolipoprotein A1 ratio was associated with the highest odds ratio for myocardial infarction among all modifiable CV risk factors [6]. The fact that elevated levels of low-density lipoprotein-cholesterol (LDL-C) play a causal role in the development of ASCVD is well accepted, given the results of numerous epidemiological and genetic 
studies, as well as randomized controlled clinical trials (RCTs) $[7-10,11 \bullet]$. The positive association between LDL-C and ASCVD extends even to very low levels of LDL-C [12]. While the overall CV risk is considerably low in populations with genetically determined low levels of LDL-C [13], lifetime exposure to elevated LDL-C is associated with an excessive risk of ASCVD, e.g., in patients with familial hyperlipoproteinemia (FH) [14].

\section{Therapeutic Approaches in the Treatment of Hypercholesterolemia}

Statins have become a primary therapeutic cornerstone in ASCVD prevention since they have been shown to reduce $\mathrm{CV}$ events by reducing levels of LDL-C [15]. But despite the proven efficacy and safety of statins, several aspects indicate the substantial need for additional or alternative LDL-C lowering therapies.

First of all, a considerably high variability in individual responses to statin therapy has been shown [16]. Furthermore, possible side effects of statin therapy such as statin associated muscle symptoms become relevant by potentially reducing adherence to treatment. Consequently, side effects of statin therapy represent a strong predictor of failure to meet LDL-C goals $[17,18]$. Observational data suggest that as many as half of patients in whom statin therapy is initiated will discontinue it within 1 year [19]. Moreover, a substantially elevated risk for $\mathrm{CV}$ events remains in a large proportion of patients, especially in those with persistent elevation of LDL-C levels despite a maximum tolerated dose of statin therapy [20].

Ezetimibe lowers LDL-C by preventing cholesterol absorption through inhibition of the Niemann-Pick C1-Like 1 protein. The results of the IMPROVE-IT trial showed that, after ACS, adding ezetimibe to statin therapy to lower LDL$\mathrm{C}$ below levels achieved by statin therapy alone is safe and further improved CV outcome [21].

\section{The Role of PCSK9 in the Treatment of Hypercholesterolemia}

In 2003, Abifadel and colleagues reported a gain of function mutation of proprotein convertase subtilisin-kexin type 9 (PCSK9) causing FH [22]. Subsequently, it was reported that sequence variations in PCSK9 with lower levels of LDL-C were associated with a reduced risk of coronary disease [23-25]. PCSK9 is synthesized and secreted from the liver and binds LDL receptors both intracellularly and extracellularly, promoting their lysosomal degradation in hepatocytes and thereby increased levels of circulating LDL [26]. Consequently, PCSK9 was considered a highly promising target for LDL-C lowering and preventive therapies [27].

\section{PCSK9 Antibody Therapy Prevents PCSK9 from Binding to the LDL Receptor}

Initially, therapeutic approaches to lower circulating levels of PCSK9 have focused on the use of monoclonal antibodies. These antibodies sequester PCSK9 in the reticuloendothelial system, preventing it to bind to the LDL receptor $[28,29]$. Recently, two large RCTs have shown that subcutaneous administration of a monoclonal PCSK9 antibody safely reduced both LDL-C levels and CV events in patients at very high CV risk [30, 31]. PCSK9 antibodies need to be administered by subcutaneous injections once or twice monthly [27].

\section{RNA-Targeted Therapy to Prevent Hepatic Synthesis of PCSK9}

siRNA molecules engage the natural pathway of RNA interference (RNAi) by binding to the RNA-induced silencing complex (RISC) intracellularly, enabling it to catalytically cleave messenger RNA (mRNA), which itself is then degraded and translation into protein is inhibited [32, 33] (Fig. 1). Previous findings have demonstrated the ability of RNAi therapeutic agents to inhibit the synthesis of liver-derived target proteins in diseases such as transthyretin amyloidosis and cancer with liver involvement $[34,35]$. Since circulating PCSK9 is largely derived from the liver, targeting its hepatic production through administration of a siRNA molecule offers an alternative to the use of monoclonal antibodies.

In a first randomized, single-blind, placebo-controlled phase 1 trial with 32 healthy volunteers, the administration of ALN-PCS, an intravenous formulation of a PCSK9 synthesis inhibiting siRNA, led to both a reduction in the levels of PCSK9 and the concentration of serum LDL-C [36].

\section{Inclisiran Is a Subcutaneously Delivered siRNA Directed Against PCSK9}

Inclisiran (ALN-PCSsc) is a long-acting, synthetic siRNA directed against PCSK9, conjugated to triantennary $\mathrm{N}$ acetylgalactosamine carbohydrates, which bind to abundant liver-expressed asialoglycoprotein receptors, leading to a rapid uptake of inclisiran specifically into hepatocytes after subcutaneous injection [37] (Fig. 1). Other than ALN-PCS (intravenous formulation), inclisiran is delivered subcutaneously.

\section{Safety, Side Effect Profile, and Pharmacodynamic Effects of Inclisiran}

Safety, side effect profile, and pharmacodynamic effects of PCSK9 siRNA were assessed in another phase 1 trial [38]. In 
this single-blind study, a total of 69 volunteers were randomized in a 3:1 ratio to receive either a subcutaneous injection of inclisiran or placebo in either a single ascending dose $(25,100$, 300,500 , or $800 \mathrm{mg}$ ) or a multiple-dose phase (125 mg weekly for four doses, $250 \mathrm{mg}$ every other week for two doses, or 300 or $500 \mathrm{mg}$ monthly for two doses, with or without concurrent statin therapy). Participants had to have an LDL-C level of at least $100 \mathrm{mg} / \mathrm{dl}$ and a fasting triglyceride level of at least $400 \mathrm{mg} / \mathrm{dl}$. Furthermore, participants with a history of CV disease, cerebrovascular disease, or diabetes were excluded, except for those on a stable statin therapy. Such patients could be enrolled if they had non-insulin-dependent diabetes mellitus or controlled hypertension. Measured 84 days after the initial injection, doses of $300 \mathrm{mg}$ inclisiran or more (in single or multiple doses) significantly reduced levels of PCSK9 and LDL-C. These reductions were maintained throughout 6 months. No serious adverse events were observed with inclisiran. All adverse events were classified as either mild or moderate and included mostly cough, musculoskeletal pain, nasopharyngitis, headache, back pain, and diarrhea.

\section{Inclisiran Lowers LDL-C Among Patients at High Cardiovascular Risk}

ORION-1 was a phase 2, dose-finding trial evaluating the efficacy of different doses and dosing intervals of inclisiran among patients at high or very high $\mathrm{CV}$ risk with elevated LDL-C levels despite receiving the maximum dose of a statin [39••]. In this randomized, double-blind, placebo-controlled trial, a total of 501 patients were randomized to receive a single dose of placebo or 200,300 , or $500 \mathrm{mg}$ of inclisiran or two doses (at day 1 and 90) of placebo or 100, 200, or $300 \mathrm{mg}$ of inclisiran. Patients had to have an LDL-C level of $70 \mathrm{mg} / \mathrm{dl}$ or higher (with a history of CVD) or $100 \mathrm{mg} / \mathrm{dl}$ or higher (without a history of CVD), respectively. When entering the study, $73 \%$ of the patients were receiving a statin, and $31 \%$ of the patients were receiving ezetimibe.

Inclisiran significantly reduced LDL-C levels measured at day 180 (primary end point) in the single-dose regimens, as well as in the two-dose regimens. LDL-C levels already declined at day 14 after the first injection with a nadir at day 60 (single dose) and day 150 (two doses), respectively. The greatest reduction of LDL-C at day 180 was achieved by the two-dose 300-mg regime (least squares mean reduction: $52.6 \%$ ). In this regime, $48 \%$ of the patients had an LDL-C below $50 \mathrm{mg} / \mathrm{dl}$ at day 180 .

All of the patients who received two 300-mg doses of inclisiran had a decline in LDL-C at day 180 (mean absolute change of $-64.2 \pm 20.7 \mathrm{mg} / \mathrm{dl}$ ). In contrast, there were large variations with regard to the changes in LDL-C at day 180 among patients receiving placebo against a background statin therapy at the maximum possible dose (mean absolute change of $-0.7 \pm 25.6 \mathrm{mg} / \mathrm{dl})$.
Adverse events were reported in $76 \%$ of the patients receiving inclisiran and $76 \%$ of the patients receiving placebo. $95 \%$ of these adverse events were either mild or moderate (mostly myalgia, headache, fatigue, nasopharyngitis, back pain, hypertension, diarrhea, and dizziness) and did not differ significantly between the groups receiving inclisiran and those receiving placebo.

Transient injection site reactions were reported in $5 \%$ of the patients who received injections of inclisiran. Elevations in Creactive protein or effects on platelet levels were not observed among patients receiving inclisiran. Transient elevations in hepatic enzyme levels were observed in three patients receiving inclisiran.

\section{Administering Inclisiran Every 6 Months Lowers LDL-C by Approximately 50\%}

Recently, two phase 3 studies, i.e., the ORION-10 and the ORION-11 trial, two randomized, double-blind, placebo-controlled, parallel-group, phase 3 trials, assessed the efficacy, safety, and adverse-event profile of inclisiran over 18 months in patients at high or very high $\mathrm{CV}$ risk with an LDL-C $\geq 70 \mathrm{mg} / \mathrm{dl}$ (in patient with ASCVD) or $\geq 100 \mathrm{mg} / \mathrm{dl}$ (in patients with an ASCVD risk equivalent) despite receiving a statin therapy at the maximum tolerated dose with or without ezetimibe [40••] (Table 1). ORION-10 was conducted in the USA and enrolled 1561 patients with ASCVD, whereas ORION-11 was conducted in Europe and South Africa and enrolled 1617 patients with ASCVD or an ASCVD risk equivalent (type 2 diabetes, FH, or a 10 -year risk of a CV event of $\geq 20 \%$ as assessed by the Framingham Risk Score for Cardiovascular Disease or equivalent). Patients were randomized in a 1:1 ratio to receive either $284 \mathrm{mg}$ of inclisiran (equivalent to $300 \mathrm{mg}$ inclisiran sodium) or placebo, administered as a 1.5-ml subcutaneous injection on day 1 , day 90, and every 6 months thereafter over a period of 540 days. The coprimary end points were the placebo-corrected percentage change in LDL-C from baseline to day 510 and the time-adjusted percentage change in LDL-C from baseline after day 90 up to day 540 .

In the ORION-10 trial, patients had a baseline LDL-C of $104.7 \pm 38.3 \mathrm{mg} / \mathrm{dl}$ and in the ORION-11 trial, patients had a baseline LDL-C of $105.5 \pm 39.1 \mathrm{mg} / \mathrm{dl}$. At day 510, inclisiran reduced LDL-C by $52.3 \%$ in the ORION-10 trial and by $49.9 \%$ in the ORION-11 trial, respectively. The corresponding time-adjusted percentage changes of LDL-C were $53.8 \%$ and $49.2 \%$, respectively.

Adverse events were reported in $73.5 \%$ of the patients receiving inclisiran and in $74.8 \%$ of the patients receiving placebo in the ORION-10 trial and in $82.7 \%$ of the patients receiving inclisiran and in $81.5 \%$ of the patients receiving placebo in the ORION-11 trial. Most of the events were reported to be mild or moderate. Furthermore, laboratory results with 


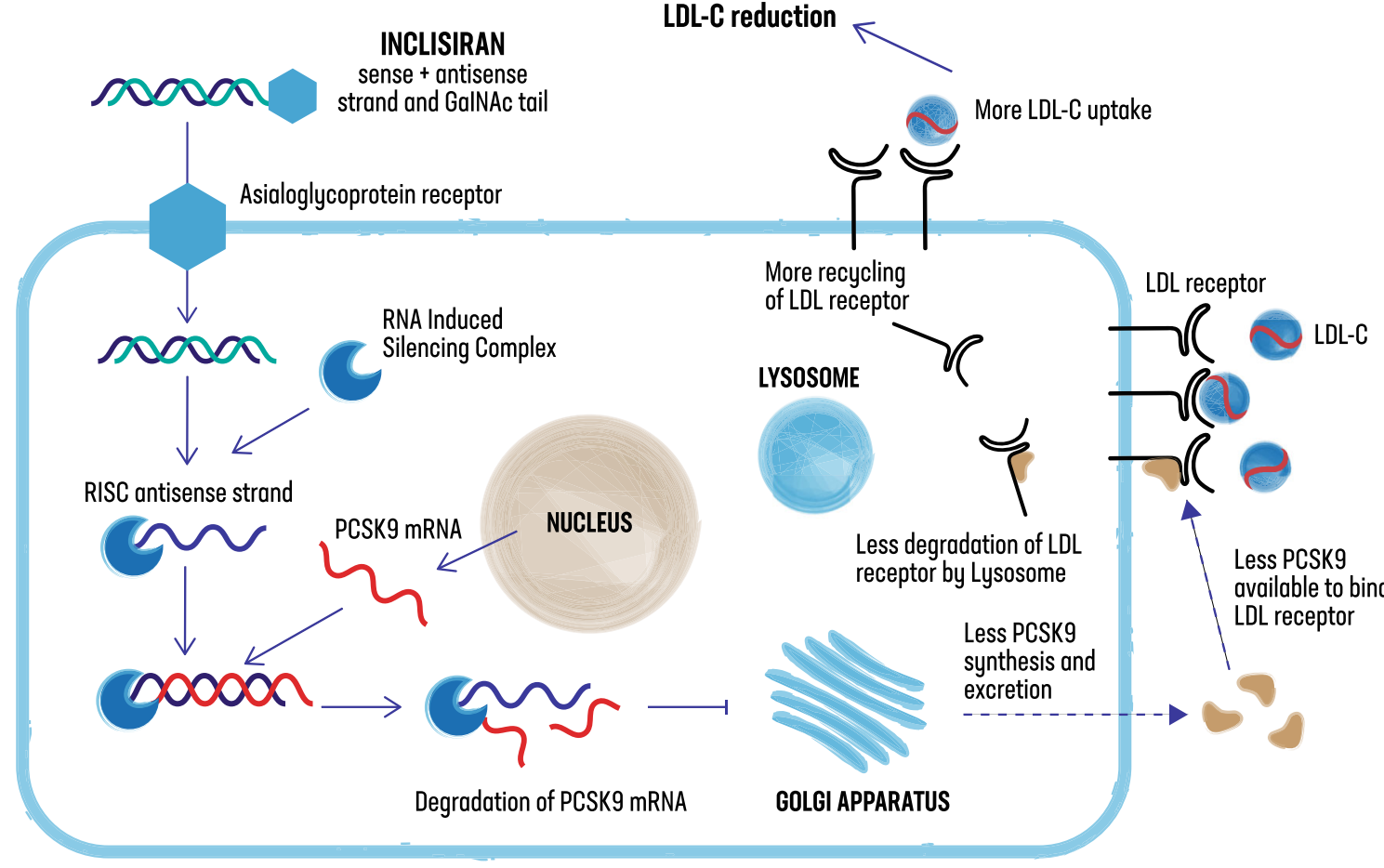

\section{HEPATOCYTE}

Fig. 1 Inclisiran: mechanism of action. Inclisiran is a long-acting, synthetic siRNA directed against proprotein convertase subtilisin-kexin type 9 (PCSK9), conjugated to triantennary $\mathrm{N}$-acetylgalactosamine carbohydrates, which bind to abundant liver-expressed asialoglycoprotein receptors, leading to a rapid uptake of inclisiran specifically into hepatocytes. Inclisiran engages the natural pathway of

respect to C-reactive protein and platelet count as well as the incidences of cancer-related deaths and new, worsening, or recurrent cancer were similar in the inclisiran and placebo groups in each trial. In the ORION-10 trial, serious adverse events were reported in $22.4 \%$ of the patients receiving inclisiran, including 12 deaths (1.5\%), and in $26.3 \%$ of the patients receiving placebo, including 11 deaths $(1.4 \%)$; in the ORION-10 trial, serious adverse events were reported in $22.3 \%$, including 14 deaths $(1.7 \%)$, of the patients receiving inclisiran and in $22.5 \%$ of the patients receiving placebo, including 15 deaths (1.9). Injection site adverse events were more frequent with inclisiran than with placebo (2.6\% vs. $0.9 \%$ in the ORION-10 trial and $4.7 \%$ vs. $0.5 \%$ in the ORION-11 trial). These reactions were generally mild and non-persistent.

A prespecified exploratory end point, which included cardiac death, any signs or symptoms of cardiac arrest, non-fatal myocardial infarction, or stroke, occurred in $7.4 \%$ in the inclisiran group and in $10.2 \%$ in the placebo group in the ORION-10 trial and in $7.8 \%$ in the inclisiran group and in $10.3 \%$ in the placebo group in the ORION-11 trial.

Recently, the results of the phase 3 ORION-9 trial demonstrated the safety and efficacy of inclisiran in patients with heterozygous FH [41] (Table 1).
RNA interference (RNAi) by binding to the RNA-induced silencing complex (RISC) intracellularly, enabling it to catalytically cleave PCSK9 messenger RNA (mRNA), which itself is then degraded, inhibiting translation into PCSK9. With less PCSK9 available, more low-density lipoprotein-cholesterol (LDL-C) receptors can be recycled to the hepatic membrane for LDL-C uptake

Furthermore, the ORION-4 trial is a dedicated ongoing CV outcome trial to examine the effect of inclisiran on cardiovascular events in patients at very high CV risk [42].

\section{Conclusions}

Statins, in addition to non-pharmaceutical lifestyle modifications, have become the first-line therapy in preventing and treating ASCVD. Consequently, current guidelines recommend that a statin should be initiated in patients with hypercholesterolemia, but many patients, especially those at high or very high $\mathrm{CV}$ risk, require additional LDL-C lowering therapies $[9,10]$. However, long-term adherence for statin therapy is suboptimal [43]. Inclisiran is a long-acting, synthetic siRNA; generally, RNAi-based therapies seem to require less frequent dosing than antibody therapies or other RNA-based therapies, such as antisense oligonucleotides [44].

The results of the phase 1 , phase 2, and phase 3 trials with inclisiran suggest that its subcutaneous administration results in LDL-C reductions of approximately $50 \%[45,46]$. In the phase 3 ORION-10 and ORION-11 trials, inclisiran reduced LDL-C levels by $52.3 \%$ and $49.9 \%$ at day 510 , respectively. These reductions were achieved on top of a maximum 
Table 1 Inclisiran: overview of phase 3 clinical trials

\begin{tabular}{lllllll}
\hline Trial name & $\begin{array}{l}\text { Number of } \\
\text { patients }\end{array}$ & Type of patients & $\begin{array}{l}\text { Baseline LDL-C } \\
\text { level (mg/dl) }\end{array}$ & $\begin{array}{l}\text { Dosing of } \\
\text { inclisiran sodium }\end{array}$ & $\begin{array}{l}\text { Reduction in plasma } \\
\text { LDL-C level }\end{array}$ & $\begin{array}{l}\text { Follow- } \\
\text { up }\end{array}$ \\
\hline ORION-10 & 1.561 & Patients with ASCVD & 105 & $300 \mathrm{mg}^{1}$ & $52 \%^{2}$ & 540 days \\
ORION-11 & 1.617 & $\begin{array}{c}\text { Patients with ASCVD or } \\
\text { ASCVD risk equivalent }\end{array}$ & 106 & $300 \mathrm{mg}^{1}$ & $50 \%^{2}$ & 540 days 40 \\
ORION-9 & 482 & Patients with heterozygous FH & 153 & $300 \mathrm{mg}^{1}$ & $40 \%^{2}$ & 540 days 41 \\
\hline
\end{tabular}

$A S C V D$ atherosclerotic cardiovascular disease, $F H$ familial hypercholesterolemia, $L D L-C$ low-density lipoprotein-cholesterol

${ }^{1}$ Subcutaneous injection at day 1 , day 90 , and every 6 months thereafter

${ }^{2}$ At day 510

tolerated, guideline-recommended statin treatment with or without ezetimibe. When compared with PCSK9 antibody therapy, the LDL-C reduction came at a lower injection burden: while PCSK9 antibodies require one- or twice monthly injections, a regimen of subcutaneous injections on day 1 , day 90 , and then every 6 months was applied in the ORION-10 and the ORION-11 trials with inclisiran [40••].

In both the ORION-10 and ORION-11 trials, the prespecified $\mathrm{CV}$ outcome end point was reported with lower frequency in the inclisiran group when compared with the placebo group, but the total number of $\mathrm{CV}$ events in 540 days of trial duration was too small to yet draw definitive conclusions about effects of inclisiran on CV outcome. This question is currently being tested in an ongoing CV outcome trial- the ORION-4 trial [42].

A theoretical concern with RNA-based therapies is a potential activation of the immune system, but elevations in C-reactive protein have not yet been observed with inclisiran [39*0]. Also, effects on platelet levels have not yet been observed in patients receiving inclisiran, in contrast to findings from studies with antisense oligonucleotides and other siRNA molecules [47, 48].

Injection site adverse events were more frequent with inclisiran than with placebo in all of the trials but were mild or moderate, did not require intervention, and were non-persistent, i.e., transient. Additional longer-term safety data will be provided by the results of ongoing open-label extension studies and the CV outcome trial [42].

In conclusion, subcutaneous administration of inclisiran, a synthetic siRNA directed against PCSK9, has been proven feasible. A regimen of injecting 300-mg inclisiran sodium at day 1 , day 90 , and every 6 months thereafter lowered LDL-C by approximately $50 \%$ in patients at high or very high $\mathrm{CV}$ risk or with heterozygous $\mathrm{FH}$, therefore representing a promising future option for optimizing the prevention of ASCVD.

Acknowledgments The authors would like to thank David Pankrath for designing the figure.

Authors' Contribution David Sinning (DS) and Ulf Landmesser (UL) contributed to the conception and design of the work. DS and UL drafted and revised the manuscript. Both authors gave final approval and agree to be accountable for all aspects of work ensuring both integrity and accuracy.
Funding Open Access funding enabled and organized by Projekt DEAL.

\section{Compliance with Ethical Standards}

Conflict of Interest David Sinning has received lecture honoraria or advisory honoraria from Amgen, Sanofi, Hexal, Daiichi-Sankyo, and Berlin-Chemie. Ulf Landmesser has received lecture or advisory honoraria from Amgen, Sanofi, Novartis, Medicines Company, Bayer, and Berlin-Chemie.

Human and Animal Rights and Informed Consent This article does not contain any studies with human or animal subjects performed by any of the authors.

Open Access This article is licensed under a Creative Commons Attribution 4.0 International License, which permits use, sharing, adaptation, distribution and reproduction in any medium or format, as long as you give appropriate credit to the original author(s) and the source, provide a link to the Creative Commons licence, and indicate if changes were made. The images or other third party material in this article are included in the article's Creative Commons licence, unless indicated otherwise in a credit line to the material. If material is not included in the article's Creative Commons licence and your intended use is not permitted by statutory regulation or exceeds the permitted use, you will need to obtain permission directly from the copyright holder. To view a copy of this licence, visit http://creativecommons.org/licenses/by/4.0/.

\section{References}

Papers of particular interest, published recently, have been highlighted as:

- Of importance

•- Of major importance

1. Ford ES, Ajani UA, Croft JB, Critchley JA, Labarthe DR, Kottke TE, et al. Explaining the decrease in U.S. deaths from coronary disease, 1980-2000. N Engl J Med. 2007;356(23):2388-98.

2. Levy D. Combating the epidemic of heart disease. JAMA. 2012;308(24):2624-5.

3. Mathers CD, Loncar D. Projections of global mortality and burden of disease from 2002 to 2030. PLoS Med. 2006;3(11):e442.

4. Townsend N, Wilson L, Bhatnagar P, Wickramasinghe K, Rayner M, Nichols M. Cardiovascular disease in Europe: epidemiological update 2016. Eur Heart J. 2016;37(42):3232-45. 
5. Weir HK, Anderson RN, Coleman King SM, Soman A, Thompson TD, Hong Y, et al. Heart disease and cancer deaths - trends and projections in the United States, 1969-2020. Prev Chronic Dis. 2016;13:E157.

6. Yusuf S, Hawken S, Ounpuu S, Dans T, Avezum A, Lanas F, et al. Effect of potentially modifiable risk factors associated with myocardial infarction in 52 countries (the INTERHEART study): casecontrol study. Lancet. 2004;364(9438):937-52.

7. Goldstein JL, Brown MS. A century of cholesterol and coronaries: from plaques to genes to statins. Cell. 2015;161(1):161-72.

8. Graham I, Cooney MT, Bradley D, Dudina A, Reiner Z. Dyslipidemias in the prevention of cardiovascular disease: risks and causality. Curr Cardiol Rep. 2012;14(6):709-20.

9. Grundy SM, Stone NJ, Bailey AL, Beam C, Birtcher KK, Blumenthal RS, et al. 2018 AHA/ACC/AACVPR/AAPA/ABC/ ACPM/ADA/AGS/APhA/ASPC/NLA/PCNA guideline on the management of blood cholesterol: a report of the American College of Cardiology/American Heart Association Task Force on clinical practice guidelines. Circulation. 2019;139(25):e1082e143.

10. Mach F, Baigent C, Catapano AL, Koskinas KC, Casula M, Badimon L, et al. 2019 ESC/EAS guidelines for the management of dyslipidaemias: lipid modification to reduce cardiovascular risk. Eur Heart J. 2020;41(1):111-88.

11. Ference BA, Ginsberg HN, Graham I, Ray KK, Packard CJ, Bruckert E, et al. Low-density lipoproteins cause atherosclerotic cardiovascular disease. 1. Evidence from genetic, epidemiologic, and clinical studies. A consensus statement from the European Atherosclerosis Society Consensus Panel. Eur Heart J. 2017;38(32):2459-72 This consensus stament summarizes the consistent evidence coming from numerous epidemiological, clinical, and genetic studies that low-density lipoprotein-cholesterol unequivocally causes cardiovascular disease.

12. Chen Z, Peto R, Collins R, MacMahon S, Lu J, Li W. Serum cholesterol concentration and coronary heart disease in population with low cholesterol concentrations. BMJ. 1991;303(6797):27682 .

13. Ference BA, Yoo W, Alesh I, Mahajan N, Mirowska KK, Mewada A, et al. Effect of long-term exposure to lower low-density lipoprotein cholesterol beginning early in life on the risk of coronary heart disease: a Mendelian randomization analysis. J Am Coll Cardiol. 2012;60(25):2631-9.

14. Austin MA, Hutter CM, Zimmern RL, Humphries SE. Familial hypercholesterolemia and coronary heart disease: a HuGE association review. Am J Epidemiol. 2004;160(5):421-9.

15. Cholesterol Treatment Trialists C, Baigent C, Blackwell L, Emberson J, Holland LE, Reith C, et al. Efficacy and safety of more intensive lowering of LDL cholesterol: a meta-analysis of data from 170,000 participants in 26 randomised trials. Lancet. 2010;376(9753):1670-81.

16. Ridker PM, Mora S, Rose L, Group JTS. Percent reduction in LDL cholesterol following high-intensity statin therapy: potential implications for guidelines and for the prescription of emerging lipidlowering agents. Eur Heart J. 2016;37(17):1373-9.

17. Munkhaugen J, Sverre E, Otterstad JE, Peersen K, Gjertsen E, Perk $\mathrm{J}$, et al. Medical and psychosocial factors and unfavourable lowdensity lipoprotein cholesterol control in coronary patients. Eur J Prev Cardiol. 2017;24(9):981-9.

18. Stroes ES, Thompson PD, Corsini A, Vladutiu GD, Raal FJ, Ray KK, et al. Statin-associated muscle symptoms: impact on statin therapy-European Atherosclerosis Society Consensus Panel Statement on Assessment, Aetiology and Management. Eur Heart J. 2015;36(17):1012-22.

19. Zhang H, Plutzky J, Skentzos S, Morrison F, Mar P, Shubina M, et al. Discontinuation of statins in routine care settings: a cohort study. Ann Intern Med. 2013;158(7):526-34.
20. Jones PH, Nair R, Thakker KM. Prevalence of dyslipidemia and lipid goal attainment in statin-treated subjects from 3 data sources: a retrospective analysis. J Am Heart Assoc. 2012;1(6):e001800.

21. Cannon CP, Blazing MA, Giugliano RP, McCagg A, White JA, Theroux $\mathrm{P}$, et al. Ezetimibe added to statin therapy after acute coronary syndromes. N Engl J Med. 2015;372(25):2387-97.

22. Abifadel M, Varret M, Rabes JP, Allard D, Ouguerram K, Devillers $\mathrm{M}$, et al. Mutations in PCSK9 cause autosomal dominant hypercholesterolemia. Nat Genet. 2003;34(2):154-6.

23. Cohen JC, Boerwinkle E, Mosley TH Jr, Hobbs HH. Sequence variations in PCSK9, low LDL, and protection against coronary heart disease. N Engl J Med. 2006;354(12):1264-72.

24. Horton JD, Cohen JC, Hobbs HH. PCSK9: a convertase that coordinates LDL catabolism. J Lipid Res. 2009;50(Suppl):S172-7.

25. Zhao Z, Tuakli-Wosornu Y, Lagace TA, Kinch L, Grishin NV, Horton JD, et al. Molecular characterization of loss-of-function mutations in PCSK9 and identification of a compound heterozygote. Am J Hum Genet. 2006;79(3):514-23.

26. Mousavi SA, Berge KE, Leren TP. The unique role of proprotein convertase subtilisin/kexin 9 in cholesterol homeostasis. J Intern Med. 2009;266(6):507-19.

27. Hooper AJ, Burnett JR. Anti-PCSK9 therapies for the treatment of hypercholesterolemia. Expert Opin Biol Ther. 2013;13(3):429-35.

28. Reyes-Soffer G, Pavlyha M, Ngai C, Thomas T, Holleran S, Ramakrishnan R, et al. Effects of PCSK9 inhibition with alirocumab on lipoprotein metabolism in healthy humans. Circulation. 2017;135(4):352-62.

29. Watts GF, Chan DC, Dent R, Somaratne R, Wasserman SM, Scott $\mathrm{R}$, et al. Factorial effects of evolocumab and atorvastatin on lipoprotein metabolism. Circulation. 2017;135(4):338-51.

30. Sabatine MS, Giugliano RP, Keech AC, Honarpour N, Wiviott SD, Murphy SA, et al. Evolocumab and clinical outcomes in patients with cardiovascular disease. N Engl J Med. 2017;376(18):1713-22.

31. Schwartz GG, Steg PG, Szarek M, Bhatt DL, Bittner VA, Diaz R, et al. Alirocumab and cardiovascular outcomes after acute coronary syndrome. N Engl J Med. 2018;379(22):2097-107.

32. Bernards R. Exploring the uses of RNAi-gene knockdown and the Nobel Prize. N Engl J Med. 2006;355(23):2391-3.

33. Carthew RW, Sontheimer EJ. Origins and mechanisms of miRNAs and siRNAs. Cell. 2009;136(4):642-55.

34. Coelho T, Adams D, Silva A, Lozeron P, Hawkins PN, Mant T, et al. Safety and efficacy of RNAi therapy for transthyretin amyloidosis. N Engl J Med. 2013;369(9):819-29.

35. Tabernero J, Shapiro GI, LoRusso PM, Cervantes A, Schwartz GK, Weiss GJ, et al. First-in-humans trial of an RNA interference therapeutic targeting VEGF and KSP in cancer patients with liver involvement. Cancer Discov. 2013;3(4):406-17.

36. Fitzgerald K, Frank-Kamenetsky M, Shulga-Morskaya S, Liebow A, Bettencourt BR, Sutherland JE, et al. Effect of an RNA interference drug on the synthesis of proprotein convertase subtilisin $/$ kexin type 9 (PCSK9) and the concentration of serum LDL cholesterol in healthy volunteers: a randomised, single-blind, placebo-controlled, phase 1 trial. Lancet. 2014;383(9911):60-8.

37. Nair JK, Willoughby JL, Chan A, Charisse K, Alam MR, Wang Q, et al. Multivalent N-acetylgalactosamine-conjugated siRNA localizes in hepatocytes and elicits robust RNAi-mediated gene silencing. J Am Chem Soc. 2014;136(49):16958-61.

38. Fitzgerald K, White S, Borodovsky A, Bettencourt BR, Strahs A, Clausen V, et al. A highly durable RNAi therapeutic inhibitor of PCSK9. N Engl J Med. 2017;376(1):41-51.

39.• Ray KK, Landmesser U, Leiter LA, Kallend D, Dufour R, Karakas $\mathrm{M}$, et al. Inclisiran in patients at high cardiovascular risk with elevated LDL cholesterol. N Engl J Med. 2017;376(15):1430-40 In this phase 2 trial, inclisiran was found to safley lower lowdensity lipoprotein-cholesterol among patients at high cardiovascular risk. 
40.• Ray KK, Wright RS, Kallend D, Koenig W, Leiter LA, Raal FJ, et al. Two phase 3 trials of inclisiran in patients with elevated LDL cholesterol. N Engl J Med. 2020;382(16):1507-19 These two phase 3 trials demonstrate low-density lipoprotein-cholesterol reductions of approximately $50 \%$ with inclisran, adminstered subcutaneously every 6 months, in patients at very high cardiovascular risk.

41. Raal FJ, Kallend D, Ray KK, Turner T, Koenig W, Wright RS, et al. Inclisiran for the treatment of heterozygous familial hypercholesterolemia. N Engl J Med. 2020;382(16):1520-30.

42. Stoekenbroek RM, Kallend D, Wijngaard PL, Kastelein JJ. Inclisiran for the treatment of cardiovascular disease: the ORION clinical development program. Futur Cardiol. 2018;14(6):433-42.

43. Choudhry NK, Fischer MA, Avorn J, Liberman JN, Schneeweiss S, Pakes J, et al. The implications of therapeutic complexity on adherence to cardiovascular medications. Arch Intern Med. 2011;171(9): 814-22.

44. Ray KK, Corral P, Morales E, Nicholls SJ. Pharmacological lipidmodification therapies for prevention of ischaemic heart disease: current and future options. Lancet. 2019;394(10199):697-708.
45. Rouleau J. Improved outcome after acute coronary syndromes with an intensive versus standard lipid-lowering regimen: results from the Pravastatin or Atorvastatin Evaluation and Infection TherapyThrombolysis in Myocardial Infarction 22 (PROVE IT-TIMI 22) trial. Am J Med. 2005;118(Suppl 12A):28-35.

46. Stein EA, Mellis S, Yancopoulos GD, Stahl N, Logan D, Smith WB, et al. Effect of a monoclonal antibody to PCSK9 on LDL cholesterol. N Engl J Med. 2012;366(12):1108-18.

47. Chi X, Gatti P, Papoian T. Safety of antisense oligonucleotide and siRNA-based therapeutics. Drug Discov Today. 2017;22(5):82333.

48. Landmesser U, Haghikia A, Leiter LA et al. Effect of inclisiran, the siRNA against PCSK9, on platelets, immune cells and immunological biomarkers - a pre-specified analysis from ORION-1. Cardiovasc Res. 2020. https://doi.org/10.1093/cvr/cvaa077. (online ahead of print)

Publisher's Note Springer Nature remains neutral with regard to jurisdictional claims in published maps and institutional affiliations. 